\title{
Review: primary prevention strategies do not delay initiation of intercourse, improve contraceptive use, or reduce pregnancies in adolescent women
}

DiCenso A, Guyatt G, Willan A, et al. Interventions to reduce unintended pregnancies among adolescents: systematic review of randomised controlled trials. BMJ 2002;324:1426-30.

\section{QUESTION: Do primary prevention strategies delay sexual intercourse, improve use of contraception, and reduce unintended pregnancies in adolescents?}

\section{Data sources}

Published and unpublished studies from 1970-2000 were identified by searching CATLINE, CINAHL, Conference Papers Index, Dissertation Abstracts Online, EMBASE/Excerpta Medica, ERIC, Medline, NTIS, POPLINE, PsycINFO, Sociological Abstracts, and the Cochrane Controlled Trials Register; handsearching 10 key journals (1993-2000); reviewing the bibliographies of retrieved articles; and contacting experts.

\section{Study selection}

Randomised controlled trials (RCTs) in any language were included if they assessed the effects of pregnancy prevention programmes for adolescents (11-18 y) on initiation of sexual intercourse, use of birth control, or unintended pregnancy. Prevention programmes included sex education classes, school based clinics, family planning clinics, and community based programmes. Studies were excluded if the interventions were offered in colleges or universities, were designed to prevent a second pregnancy, or if outcomes were only knowledge and attitudes, only condom use, or only births.

\section{Data extraction}

Data were extracted on setting, participants, unit of randomisation and analysis, theoretical framework, intervention, outcomes, length of follow up, proportion followed up, and findings at last follow up by gender. Methodological quality of individual studies was assessed.

\section{Main results}

22 reports of 26 RCTs met the selection criteria (17 published, 5 unpublished). 10 studies randomised clusters such as classrooms, schools, agencies, or neighbourhoods rather than individuals. 18 studies had poor quality ( $\leq 2$ out of 4 on the modified Jadad scale). Meta-analyses using a random effects model showed that primary prevention programmes did not delay initiation of sexual intercourse in women or men (table), did not improve use of contraception at every intercourse or at last intercourse in women or men (table), did not reduce pregnancy rates in women, and actually increased reported pregnancies in partners of men (table).

\section{Conclusion}

No primary prevention strategies evaluated to date delay initiation of sexual intercourse or improve contraceptive use in adolescent women and men, or reduce pregnancies in young women.
Sources of funding:

National Health Research Development Program, Health Canada; Ontario Ministry of Health and Long-Term Care; Region of

Hamilton-Wentworth Social and Public Health Services PHRED Program.

For correspondence: Dr A DiCenso, School of Nursing, McMaster University, Hamilton, Ontario, Canada. dicensoa@mcmaster.ca

Abstract also appears in Evidence-Based

Medicine

\section{COMMENTARY}

Unintended pregnancy in adolescence continues to be an important social and public health concern in industrialised countries. The conclusions of the meta-analysis by DiCenso $e t$ al raise important questions about the efficacy of primary prevention strategies to delay initiation of sexual intercourse, improve contraceptive use, and reduce unintended pregnancy in youth. The conclusions are discouraging and may be somewhat misleading because of methodological problems in the studies reviewed.

The review of published and unpublished studies was comprehensive in that it covered a long time period (1970-2000); most of the studies were done in the 1990s. The authors reviewed controlled trials of interventions for a wide age range of teens, with ages ranging from 11-18 years. It would have been interesting to see if the effectiveness of the interventions differed by the age of the participants. 18 of the 26 studies were of poor methodological quality based on criteria of appropriateness of randomisation, bias in data collection, adequacy of follow up, and attrition rates in comparison groups.

The authors note that in 21 of 26 studies reviewed, the control groups received another intervention that could have affected the study outcomes. This point is crucial to understanding the results and could attenuate the findings of the meta-analysis. Clearly, the control interventions and the experimental interventions may both be superior to no intervention.

What can be done to prevent early sexual activity and unintended pregnancy in adolescents? The results of this review of current pregnancy prevention interventions suggest that we do not fully understand the phenomenon of adolescent sexual behaviour nor have we identified consistently effective ways to change it. As DiCenso et al suggest, development of future interventions needs to address the methodological limitations of previous studies and incorporate input from adolescents themselves. Lynn Rew, RNC, EdD, FAAN Denton E Louise Cooley and Family Centennial Professor in Nursing The University of Texas at Austin, Austin, Texas, USA

Primary prevention strategy $v$ control (alternate intervention or nothing) for adolescents*

\begin{tabular}{|c|c|c|c|c|}
\hline \multirow[b]{2}{*}{ Outcomes } & \multirow[b]{2}{*}{ Number of trials } & \multicolumn{2}{|l|}{ Weighted event rates } & \multirow[b]{2}{*}{ Pooled odds ratio (OR) $(95 \% \mathrm{Cl}) \dagger$} \\
\hline & & Primary prevention & Control & \\
\hline \multicolumn{5}{|l|}{ Women } \\
\hline Delayed initiation of intercourse & $13(n=9642)$ & $22 \%$ & $20 \%$ & $1.12(0.96$ to 1.30$)$ \\
\hline Used contraception at every intercourse & $8(n=1967)$ & $45 \%$ & $44 \%$ & 0.95 (0.69 to 1.30$)$ \\
\hline Used contraception at last intercourse & $5(n=799)$ & $50 \%$ & $46 \%$ & 1.05 (0.50 to 2.19$) \ddagger$ \\
\hline Pregnancy & $12(n=8019)$ & $4.4 \%$ & $4.8 \%$ & $1.04(0.78$ to 1.40$)$ \\
\hline \multicolumn{5}{|l|}{ Men } \\
\hline Delayed initiation of intercourse & $11(\mathrm{n}=7418)$ & $29 \%$ & $30 \%$ & $0.99(0.84$ to 1.16$)$ \\
\hline Used contraception at every intercourse & $3(n=1505)$ & $36 \%$ & $38 \%$ & $0.90(0.70$ to 1.16$)$ \\
\hline Used contraception at last intercourse & $4(n=1262)$ & $69 \%$ & $64 \%$ & 1.25 (0.99 to 1.59$)$ \\
\hline Pregnancy in partners & $5(n=3759)$ & $3.4 \%$ & $2.4 \%$ & 1.54 (1.03 to 2.29$) \S$ \\
\hline
\end{tabular}

*Abbreviations defined in glossary. Weighted event rates provided by author. Follow up ranged from 2-54 months.

†For intercourse and pregnancy outcomes, OR $<1.0$ favours intervention; for contraception outcomes, OR $>1$ favours intervention. $¥$ Significant heterogeneity among trials. §Statistically significant. 